Turk. J. Math. Comput. Sci.

13(2)(2021) 234-238

(C) MatDer

DOI : $10.47000 /$ tjmcs. 783597

\title{
Some Divisibility Properties of Lucas Numbers
}

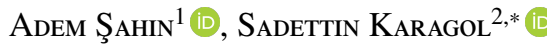 \\ ${ }^{1}$ Department of Mathematics and Science Education, Faculty of Education, Tokat Gaziosmanpasa University, Tokat, Turkey. \\ ${ }^{2}$ M. Emin Saraç Anatolian Religious Vocational High School, Tokat, Turkey.
}

Received: 21-08-2020 • Accepted: 18-08-2021

Aвstract. The Lucas number sequence is a popular number sequence that has been described as similar to the Fibonacci number sequence. A lot of research has been done on this number sequence. Some of these studies are on the divisibility properties of this number sequence. Carlitz (1964) examined the requirement that a given Lucas number can be divided by another Lucas number. After that, many studies have been done on this subject. In the present article, we obtain some divisibility properties of the Lucas Numbers. First, we examine the case $L_{(2 n-1) m} / L_{m}$ and then we obtain $L_{(2 n-1) m}$ using different forms of Lucas numbers.

2010 AMS Classification: 11A05, 11A07

Keywords: Lucas sequence, divisibility, recurrence relation.

\section{INTRODUCTION}

$L_{n} \mathrm{n}$ th term of Lucas sequence with $L_{0}=2, L_{1}=1$, and $L_{n+1}=L_{n}+L_{n-1}, n>0$. The first few Lucas numbers are $2,1,3,4,7,11,18,29,47,76,123,199,322, \ldots$

Let $\alpha=\frac{1+\sqrt{5}}{2}$ and $\beta=\frac{1-\sqrt{5}}{2}$ are the roots of the quadratic equation $x^{2}-x-1=0$, then $L_{n}=\alpha^{n}+\beta^{n}$. It is obvious that $\alpha+\beta=1, \alpha \cdot \beta=-1, \alpha^{2}=\alpha+1$ and $\beta^{2}=\beta+1$.

Many studies have been conducted on the divisibility properties of Lucas numbers. Carlitz [1] proved that " $L_{m}$ । $L_{n}$ $\Rightarrow n=(2 k-1) m, m>1$ ". Carlitz and Hunter [2] have reached the following equations;

$$
\begin{aligned}
L_{n-1}^{4}+L_{n}^{4}+L_{n+1}^{4} & =2\left[2 \cdot L_{n}^{2}-5(-1)^{n}\right]^{2}, \\
L_{n+1}^{5}-L_{n}^{5}-L_{n-1}^{5} & =5 L_{n+1} L_{n} L_{n-1}\left[2 \cdot L_{n}^{2}-5(-1)^{n}\right], \\
L_{n+1}^{7}-L_{n}^{7}-L_{n-1}^{7} & =7 L_{n+1} L_{n} L_{n-1}\left[2 \cdot L_{n}^{2}-5(-1)^{n}\right]^{2} .
\end{aligned}
$$

Also, Carlitz [3] obtained $\left\lfloor\alpha^{k} L_{n}+\frac{1}{2}\right\rfloor=L_{n+k}, n \geq k+2, k \geq 2$.

*Corresponding Author

Email addresses: adem.sahin@gop.edu.tr (A. Sahin), karagolsadettin@gmail.com (S. Karagol) 
Hoggatt [5] achieved the following equations in his work titled Lucas triangle;

$$
\begin{aligned}
L_{n}^{1}= & L_{n}, \\
L_{n}^{2}= & L_{2 n}+2(-1)^{n}, \\
& \vdots \\
L_{n}^{8}= & L_{8 n}+8(-1)^{n} L_{n}^{6}-20 \cdot L_{n}^{4}+16(-1)^{n} L_{n}^{2}-2 .
\end{aligned}
$$

Vajda [11] has reached many equations regarding Lucas Numbers. Some of these are

$i$. Let $t$ odd prime, $L_{k t}=L_{t}^{k}+\sum_{i=1}^{\lfloor k / 2\rfloor} \frac{k}{i} \cdot(-1)^{i(t+1)} \cdot L_{t}{ }^{k-2 i}\left(\begin{array}{c}k-i-1 \\ i-1\end{array}\right)$,

ii. If $(s, t)=d$, than $\left(L_{s}, L_{t}\right)=L_{d}$,

iii. $L_{n+r}+(-1)^{r} \cdot L_{n-r}=L_{r} \cdot L_{n}$,

iv. $\left(L_{n}\right)^{2}=L_{2 n}+(-1)^{n} .2$,

$v$. If $p$ is prime, than $L_{p} \equiv 1(\bmod p)$.

Koshy has done some studies on Lucas numbers. Some of these are given below:

i. [8] $L_{2 m+2 n}+L_{2 m-2 n}=L_{2 m} \cdot L_{2 n}$ and $L_{n+r}^{2}+L_{n-r}^{2}=L_{2 n} \cdot L_{2 r}+4 .(-1)^{n+r}$,

ii. [9] $L_{k}^{2}+L_{k+1}^{2}=L_{2 k}+L_{2 k+2}, L_{n+1}^{3}+L_{n}^{3}-L_{n-1}^{3}=5 L_{3 n}, L_{m+r} \cdot L_{m+r+1}+L_{m-r} \cdot L_{m-r+1}=L_{2 m+2 r+1}+L_{2 m-2 r+1}+2 \cdot(-1)^{m+r}$ and $L_{m+r} \cdot L_{m+r+1}+L_{m-r} \cdot L_{m-r+1}=L_{2 m+1}+L_{2 r}+2 \cdot(-1)^{m+r}$,

iii. [10] $L_{n+1}^{2}-L_{n}^{2}=L_{n-1} \cdot L_{n+2}, L_{3 n}=L_{n}$. $\left[L_{2 n}-(-1)^{n}\right]$ and $L_{r-1} \cdot L_{r+1}-L_{r}^{2}=5 .(-1)^{r-1}, r \geq 1$.

In addition, Hoggatt and Bergum [6] examined the divisibility and congruance relationships in Lucas numbers. Keskin and Bitim [7] studied on Fibonacci and Lucas congruences and obtained divisibility properties of Fibonacci and Lucas numbers. In [4] authors obtained congruences including Fibonacci and Lucas numbers.

\section{Some Divisibility Properties of Lucas Numbers}

Theorem 2.1. Let $m$ is a naturel number, then

$$
L_{5 m} / L_{m}=\left\{\begin{array}{l}
\left(L_{3 m} / L_{m}\right)^{2}-\left(L_{3 m} / L_{m}\right)-1, \quad m \text { is odd } \\
\left(L_{3 m} / L_{m}\right)^{2}+\left(L_{3 m} / L_{m}\right)-1, \quad m \text { is even }
\end{array}\right.
$$

Proof. Let $m$ be odd and consider the Binet's formula $L_{n}=\alpha^{n}+\beta^{n}$, then

$$
\begin{aligned}
\left(L_{3 m} / L_{m}\right)^{2}-\left(L_{3 m} / L_{m}\right)-1 & =\left(\frac{\alpha^{3 m}+\beta^{3 m}}{\alpha^{m}+\beta^{m}}\right)^{2}-\left(\frac{\alpha^{3 m}+\beta^{3 m}}{\alpha^{m}+\beta^{m}}\right)-1 \\
& =\left(\left(\alpha^{2 m}+\beta^{2 m}\right)+1\right)^{2}-\left(\alpha^{2 m}+\beta^{2 m}+1\right)-1 \\
& =\alpha^{4 m}-(\alpha \cdot \beta)^{m} \cdot \alpha^{2 m}-(\alpha \cdot \beta)^{m} \cdot \beta^{2 m}+\alpha^{2 m} \cdot \beta^{2 m}+\beta^{4 m} \\
& =\frac{\left(\alpha^{m}+\beta^{m}\right)\left(\alpha^{4 m}-\alpha^{3 m} \cdot \beta^{m}+\alpha^{2 m} \cdot \beta^{2 m}-\alpha^{m} \cdot \beta^{3 m}+\beta^{4 m}\right)}{\left(\alpha^{m}+\beta^{m}\right)} \\
& =L_{5 m} / L_{m} .
\end{aligned}
$$

The proof is similar for $m$ is even.

Theorem 2.2. Let $m, n$ are naturel numbers, for $n \geq 3$, then

$$
\left(L_{(2 n-1) m} / L_{m}\right)^{2}= \begin{cases}\left(L_{(2 n-3) m} / L_{m}\right) \cdot\left(L_{(2 n+1) m} / L_{m}\right)+\left(L_{3 m} / L_{m}\right)+1, & \text { m is odd } \\ \left(L_{(2 n-3) m} / L_{m}\right) \cdot\left(L_{(2 n+1) m} / L_{m}\right)-\left(L_{3 m} / L_{m}\right)+1, & \text { m is even } .\end{cases}
$$


Proof. Let $m$ be even and consider the Binet's formula $L_{n}=\alpha^{n}+\beta^{n}$, then

$$
\begin{aligned}
& \left(L_{(2 n-3) m} / L_{m}\right) \cdot\left(L_{(2 n+1) m} / L_{m}\right)-\left(L_{3 m} / L_{m}\right)+1 \\
= & \left(\frac{\alpha^{(2 n-3) m}+\beta^{(2 n-3) m}}{\alpha^{m}+\beta^{m}}\right) \cdot\left(\frac{\alpha^{(2 n+1) m}+\beta^{(2 n+1) m}}{\alpha^{m}+\beta^{m}}\right)-\left(\frac{\alpha^{3 m}+\beta^{3 m}}{\alpha^{m}+\beta^{m}}\right)+1 \\
= & \frac{\alpha^{(2 n-1) 2 m}+(\alpha \cdot \beta)^{2 m n} \cdot\left(\alpha^{-3 m} \cdot \beta^{m}+\alpha^{m} \cdot \beta^{-3 m}\right)+\beta^{(2 n-1) 2 m}-\alpha^{4 m}}{\left(\alpha^{m}+\beta^{m}\right)\left(\alpha^{m}+\beta^{m}\right)} \\
& +\frac{-\alpha^{m} \cdot \beta^{3 m}-\beta^{m} \cdot \alpha^{3 m}-\beta^{4 m}+\alpha^{2 m}+2 \cdot(\alpha \cdot \beta)^{m}+\beta^{2 m}}{\left(\alpha^{m}+\beta^{m}\right)\left(\alpha^{m}+\beta^{m}\right)} \\
= & \frac{\alpha^{(2 n-1) 2 m}+\beta^{4 m}+\alpha^{4 m}+\beta^{(2 n-1) 2 m}-\alpha^{4 m}-\beta^{2 m}-\alpha^{2 m}-\beta^{4 m}+\alpha^{2 m}+2+\beta^{2 m}}{\left(\alpha^{m}+\beta^{m}\right)\left(\alpha^{m}+\beta^{m}\right)} \\
= & \frac{\alpha^{(2 n-1) 2 m}+2+\beta^{(2 n-1) 2 m}}{\left(\alpha^{m}+\beta^{m}\right)\left(\alpha^{m}+\beta^{m}\right)} \\
= & \frac{\alpha^{(2 n-1) 2 m}+2(\alpha \cdot \beta)^{(2 n-1) m}+\beta^{(2 n-1) 2 m}}{\left(\alpha^{m}+\beta^{m n}\right)\left(\alpha^{m}+\beta^{m}\right)} \\
= & \frac{\left(\alpha^{(2 n-1) m}+\beta^{(2 n-1) m}\right)^{2}}{\left(\alpha^{m}+\beta^{m}\right)^{2}} \\
= & \left(L_{(2 n-1) m} / L_{m}\right)^{2} .
\end{aligned}
$$

The proof is similar for $m$ is odd.

Theorem 2.3. Let $m>3$ and $n \geq 4$ are naturel numbers, then

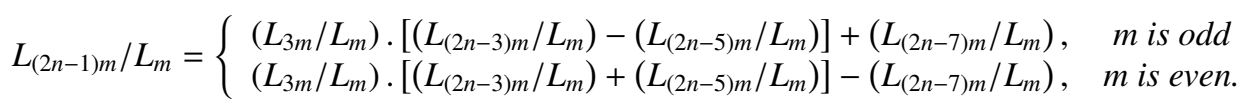

Proof. Let $m$ be odd and consider the Binet's formula $L_{n}=\alpha^{n}+\beta^{n}$, then

$$
\begin{aligned}
& \left(L_{3 m} / L_{m}\right) \cdot\left[\left(L_{(2 n-3) m} / L_{m}\right)-\left(L_{(2 n-5) m} / L_{m}\right)\right]+\left(L_{(2 n-7) m} / L_{m}\right) \\
= & \left(\frac{\alpha^{3 m}+\beta^{3 m}}{\alpha^{m}+\beta^{m}}\right) \cdot\left[\frac{\alpha^{(2 n-3) m}+\beta^{(2 n-3) m}-\alpha^{(2 n-5) m}-\beta^{(2 n-5) m}}{\alpha^{m}+\beta^{m}}\right]+\left(\frac{\alpha^{(2 n-7) m}+\beta^{(2 n-7) m}}{\alpha^{m}+\beta^{m}}\right) \\
= & \frac{\alpha^{2 n m}+\alpha^{3 m} \cdot \beta^{(2 n-3) m}-\alpha^{(2 n-2) m}-\alpha^{3 m} \cdot \beta^{(2 n-5) m}+\beta^{3 m} \cdot \alpha^{(2 n-3) m}+\beta^{2 n m}}{\left(\alpha^{m}+\beta^{m}\right) \cdot\left(\alpha^{m}+\beta^{m}\right)} \\
& +\frac{-\beta^{3 m} \cdot \alpha^{(2 n-5) m}-\beta^{(2 n-2) m}+\alpha^{(2 n-6) m}+\alpha^{m} \cdot \beta^{(2 n-7) m}+\beta^{m} \cdot \alpha^{(2 n-7) m}+\beta^{(2 n-6) m}}{\left(\alpha^{m}+\beta^{m}\right) \cdot\left(\alpha^{m}+\beta^{m}\right)} \\
= & \frac{\alpha^{2 n m}+\beta^{2 n m}+\alpha^{2 n m} \cdot \alpha^{-7 m} \cdot\left(\beta^{3 m} \cdot \alpha^{4 m}-\beta^{3 m} \cdot \alpha^{2 m}-\alpha^{5 m}+\beta^{m}+\alpha^{m}\right)}{\left(\alpha^{m}+\beta^{m}\right) \cdot\left(\alpha^{m}+\beta^{m}\right)} \\
& +\frac{\beta^{2 n m} \cdot \beta^{-7 m} \cdot\left(\alpha^{3 m} \cdot \beta^{4 m}-\alpha^{3 m} \cdot \beta^{2 m}-\beta^{5 m}+\alpha^{m}+\beta^{m}\right)}{\left(\alpha^{m}+\beta^{m}\right) \cdot\left(\alpha^{m}+\beta^{m}\right)} \\
= & \frac{\alpha^{2 n m}+\beta^{2 n m}+\alpha^{2 n m} \cdot \alpha^{-7 m} \cdot\left(-\alpha^{5 m}\right)+\beta^{2 n m} \cdot \beta^{-7 m} \cdot\left(-\beta^{5 m}\right)}{\left(\alpha^{m}+\beta^{m}\right) \cdot\left(\alpha^{m}+\beta^{m}\right)} \\
= & \frac{\alpha^{2 n m}+(\alpha \cdot \beta)^{m} \cdot \alpha^{2 n m} \cdot \alpha^{-2 m}+\beta^{2 n m}+(\alpha \cdot \beta)^{m} \cdot \beta^{2 n m} \cdot \beta^{-2 m}}{\left(\alpha^{m}+\beta^{m}\right) \cdot\left(\alpha^{m}+\beta^{m}\right)} \\
= & \frac{\alpha^{2 n m} \cdot \alpha^{-m} \cdot\left(\alpha^{m}+\beta^{m}\right)+\beta^{2 n m} \cdot \beta^{-m} \cdot\left(\alpha^{m}+\beta^{m}\right)}{\left(\alpha^{m}+\beta^{m}\right) \cdot\left(\alpha^{m}+\beta^{m}\right)} \\
= & \frac{\alpha^{(2 n-1) m}+\beta^{(2 n-1) m}}{\alpha^{m}+\beta^{m}} \\
= & L_{(2 n-1) m} / L_{m} \cdot
\end{aligned}
$$


The proof is similar for $m$ is even.

Theorem 2.4. Let $m, n$ are naturel number, $n \geq 3$, then

$$
L_{(2 n-1) m}= \begin{cases}\left(L_{m}^{2}+2\right) \cdot L_{(2 n-3) m}-L_{(2 n-5) m}, & m \text { is odd } \\ \left(L_{m}^{2}-2\right) \cdot L_{(2 n-3) m}-L_{(2 n-5) m}, & m \text { is even } .\end{cases}
$$

Proof. Let $m$ be even and consider the Binet's formula $L_{n}=\alpha^{n}+\beta^{n}$, then

$$
\begin{aligned}
& \left(L_{m}^{2}-2\right) \cdot L_{(2 n-3) m}-L_{(2 n-5) m} \\
= & {\left[\left(\alpha^{m}+\beta^{m}\right)^{2}-2\right] \cdot\left(\alpha^{(2 n-3) m}+\beta^{(2 n-3) m}\right)-\alpha^{(2 n-5) m}-\beta^{(2 n-5) m} } \\
= & \alpha^{(2 n-1) m}+\alpha^{2 m} \cdot \beta^{(2 n-3) m}+\beta^{2 m} \cdot \alpha^{(2 n-3) m}+\beta^{(2 n-1) m}-\alpha^{(2 n-5) m}-\beta^{(2 n-5) m} \\
= & \alpha^{(2 n-1) m}+\beta^{(2 n-1) m}+\alpha^{2 n m} \cdot \alpha^{-5 m} \cdot\left[(\alpha \cdot \beta)^{2 m}-1\right]+\beta^{2 n m} \cdot \beta^{-5 m} \cdot\left[(\alpha \cdot \beta)^{2 m}-1\right] \\
= & \alpha^{(2 n-1) m}+\beta^{(2 n-1) m}=L_{(2 n-1) m} .
\end{aligned}
$$

The proof is similar for $m$ is odd.

Theorem 2.5. Let $m, n$ are naturel number, then

$$
L_{(2 n+1) m}=\left\{\begin{array}{cc}
\sum_{r=1}^{n+1} \frac{2 n+1}{2 n-2 r+3}\left(\begin{array}{c}
2 n-r+1 \\
r-1
\end{array}\right) \cdot L_{m}^{2 n-2 r+3}, & m \text { is odd }, \\
\sum_{r=1}^{n+1} \frac{2 n+1}{2 n-2 r+3}\left(\begin{array}{c}
2 n-r+1 \\
r-1
\end{array}\right) \cdot L_{m}^{2 n-2 r+3} \cdot(-1)^{r+1}, & m \text { is even } .
\end{array}\right.
$$

Proof. Let $m$ be odd.

The statement is true for $n=1$ and $n=2$.

Assume the correctness of the statement for $n=k-1$ and for $n=k$, and prove the statement for $n=k+1$.

By using Theorem 2.4 when $m$ is odd and assumption, we obtain

$$
\begin{aligned}
L_{(2 k+3) m}= & \left(L_{m}^{2}+2\right)\left[\sum_{r=1}^{k+1} \frac{2 k+1}{2 k-2 r+3}\left(\begin{array}{c}
2 k-r+1 \\
r-1
\end{array}\right) \cdot L_{m}^{2 k-2 r+3}\right] \\
& -\sum_{r=1}^{k} \frac{2 k-1}{2 k-2 r+1}\left(\begin{array}{c}
2 k-r-1 \\
r-1
\end{array}\right) \cdot L_{m}^{2 k-2 r+1} \\
= & \sum_{r=1}^{k+1} \frac{2 k+1}{2 k-2 r+3}\left(\begin{array}{c}
2 k-r+1 \\
r-1
\end{array}\right) \cdot L_{m}^{2 k-2 r+5} \\
& +\sum_{r=1}^{k+1} \frac{4 k+2}{2 k-2 r+3}\left(\begin{array}{c}
2 k-r+1 \\
r-1
\end{array}\right) \cdot L_{m}^{2 k-2 r+3} \\
& -\sum_{r=1}^{k} \frac{2 k-1}{2 k-2 r+1}\left(\begin{array}{c}
2 k-r-1 \\
r-1
\end{array}\right) \cdot L_{m}^{2 k-2 r+1} \\
= & L_{m}^{2 k+3}+(2 k+1) \cdot L_{m}^{2 k+1}+\sum_{r=1}^{k-1} \frac{2 k+1}{2 k-2 r-1}\left(\begin{array}{c}
2 k-r-1 \\
r+1
\end{array}\right) \cdot L_{m}^{2 k-2 r+1} \\
& +2 \cdot L_{m}^{2 k+1}+(4 k+2) \cdot L_{m}+\sum_{r=1}^{k-1} \frac{4 k+2}{2 k-2 r+1}\left(\begin{array}{c}
2 k-r \\
r
\end{array}\right) \cdot L_{m}^{2 k-2 r+1} \\
& -(2 k-1) \cdot L_{m}-\sum_{r=1}^{k-1} \frac{2 k-1}{2 k-2 r+1}\left(\begin{array}{c}
2 k-r-1 \\
r-1
\end{array}\right) \cdot L_{m}^{2 k-2 r+1}
\end{aligned}
$$




$$
\begin{aligned}
= & L_{m}^{2 k+3}+(2 k+3) \cdot L_{m}^{2 k+1}+(2 k+3) \cdot L_{m} \\
& +\sum_{r=1}^{k-1}\left(\begin{array}{c}
2 k-r+1 \\
r+1
\end{array}\right) \cdot L_{m}^{2 k-2 r+1} \cdot\left(\begin{array}{c}
\frac{2 \cdot(2 k+1) \cdot(k-r)}{(2 k-r) \cdot(2 k-r+1)}+ \\
\frac{2 \cdot(2 k+1) \cdot(r+1)}{(2 k-2 r+1) \cdot(2 k-r+1)}-\frac{r-1) \cdot(r+1)}{(2 k-r)(2 k-2 r+1)(2 k-r+1)}
\end{array}\right) \\
= & L_{m}^{2 k+3}+(2 k+3) \cdot L_{m}^{2 k+1}+(2 k+3) \cdot L_{m} \\
& +\sum_{r=1}^{k-1}\left(\begin{array}{c}
2 k-r+1 \\
r+1
\end{array}\right) \cdot L_{m}^{2 k-2 r+1} \cdot \frac{2 k+3}{2 k-2 r+1} \\
= & \sum_{r=1}^{k+2} \frac{2 k+3}{2 k-2 r+5}\left(\begin{array}{c}
2 k-r+3 \\
r-1
\end{array}\right) \cdot L_{m}^{2 k-2 r+5} .
\end{aligned}
$$

The proof is similar for $m$ is even.

\section{CONFLICTS OF INTEREST}

The authors declare that there are no conflicts of interest regarding the publication of this article.

\section{Authors Contribution Statement}

All authors have contributed sufficiently in the planning, execution, or analysis of this study to be included as authors. All authors have read and agreed to the published version of the manuscript.

\section{REFERENCES}

[1] Carlitz, L., A Note On Fibonacci numbers, Fibonacci Quarterly, 2(1)(1964), 15-28.

[2] Carlitz, L., Hunter, J.A.H., Sum of powers of Fibonacci and Lucas numbers, Fibonacci Quarterly, 7(5)(1969), 467-473.

[3] Carlitz, L., A Conjecture concerning Lucas numbers, Fibonacci Quarterly, 10(5)(1972), 526-550.

[4] Di Porto, A., Filipponi, P., A Probabilistic Primality Test Based on the Properties of Certain Generalized Lucas Numbers. In: Barstow D. et al. (eds) Advances in Cryptology, EUROCRYPT 88. EUROCRYPT 1988. Lecture Notes in Computer Science, vol 330. Springer, Berlin, Heidelberg, 1988.

[5] Hoggatt Jr., V.E., An application of the Lucas triangle, Fibonacci Quarterly, 8(4)(1970), 360-364.

[6] Hoggatt Jr., V.E., Bergum, G.E., Divisibility and congruence relations, Fibonacci Quarterly, 12(2)(1974), 189-195.

[7] Keskin, R., Demirturk Bitim, B., Fibonacci and Lucas congruences and their applications, Acta Math. Sinica, 27(4)(2011),725-736.

[8] Koshy, T., New Fibonacci and Lucas identities, The Mathematical Gazette, 82(495)(1998), 481-484.

[9] Koshy, T., The convergence of a Lucas series, The Mathematical Gazette, 83(497)(1999), 272-274.

[10] Koshy, T., Fibonacci and Lucas Numbers With Aplications. John Wiley \& Sons Inc., New York, USA, 2001.

[11] Vajda, S., Fibonacci and Lucas Numbers and The Golden Section, Ellis Horwood Limited, Chichester, England, 1989. 